\title{
A Novel Loss-of-Function GRN Mutation p.(Tyr229*) : Clinical and Neuropathological Features
}

Kuuluvainen, Liina

2017

Kuuluvainen , L , Pöyhönen, M , Pasanen , P , Siitonen , M , Rummukainen , J , Tienari , P J , Paetau , A \& Myllykangas , L 2017 , ' A Novel Loss-of-Function GRN Mutation p. $\left(\right.$ Tyr229* ${ }^{*}$ : Clinical and Neuropathological Features ' , Journal of Alzheimer's Disease , vol. 55 , no. 3 , pp. 1167-1174 . https://doi.org/10.3233/JAD-160647

http://hdl.handle.net/10138/311411

https://doi.org/10.3233/JAD-160647

acceptedVersion

Downloaded from Helda, University of Helsinki institutional repository.

This is an electronic reprint of the original article.

This reprint may differ from the original in pagination and typographic detail.

Please cite the original version. 
A novel loss-of-function $G R N$ mutation p.(Tyr229*): clinical and neuropathological features

Running title: Features of a novel GRN mutation

Liina Kuuluvainen, Department of Clinical Genetics, Helsinki University Central Hospital and Department of Medical Genetics, University of Helsinki, Helsinki, Finland.

Minna Pöyhönen Department of Clinical Genetics, Helsinki University Central Hospital and Department of Medical Genetics, University of Helsinki, Helsinki, Finland.

Petra Pasanen Department of Medical Biochemistry and Genetics, Institute of Biomedicine, University of Turku, and Tyks Microbiology and Genetics, Department of Medical Genetics, Turku University Hospital, Turku, Finland

Maija Siitonen Department of Medical Biochemistry and Genetics, Institute of Biomedicine, University of Turku, Turku, Finland and Department of Medical Genetics, University of Helsinki, Helsinki, Finland

Jaana Rummukainen Department of Pathology, Kuopio University Hospital, Kuopio, Finland Pentti J. Tienari, Department of Neurology, Helsinki University Hospital, and Molecular Neurology, Research Programs Unit, Biomedicum, University of Helsinki, Helsinki, Finland. Anders Paetau Department of Pathology, University of Helsinki, Helsinki, Finland Liisa Myllykangas Department of Pathology, University of Helsinki, Helsinki, Finland Corresponding author Liisa Myllykangas, fax $+358-294126700$ tel. $+358-504482805$ email.Liisa.myllykangas@helsinki.fi 


\begin{abstract}
Mutations in the progranulin $(G R N)$ gene represent about 5-10\% of frontotemporal lobar degeneration (FTLD). We describe a proband with a novel GRN mutation c.687T>A, p.(Tyr229*), presenting with dyspraxia, dysgraphia and dysphasia at the age of 60 and a very severe FTLD neuropathological phenotype with TDP43 inclusions. The nephew of the proband had signs of dementia and personality changes at the age of 60 and showed similar but milder FTLD pathology. Three other family members had had early-onset dementia. Gene expression studies showed decreased $G R N$ gene expression in mutation carriers' blood samples. In conclusion, we describe a novel GRN, p.(Tyr229*) mutation, resulting in haploinsufficiency of GRN and a severe neuropathologic FTLD phenotype.
\end{abstract}

\title{
Keywords
}

frontotemporal dementia, frontotemporal lobar degeneration, mutation, progranulin (GRN), TDP-43

\section{Introduction}

Frontotemporal lobar degeneration (FTLD) is the second most common cause of dementia in patients under the age of 65 [1] and accounts for about 5-10\% of all dementia patients [2,3]. It is a group of neurodegenerative disorders, which primarily affect the frontal and temporal cortex either symmetrically or asymmetrically. Clinically there are three disease manifestations: behavioral variant frontotemporal dementia (bvFTD), semantic dementia (SD) and progressive non-fluent aphasia (PNFA) the latter two presenting with symptoms affecting primarily language. Some patients also have significant motor symptoms including 
parkinsonism. Nearly $40-50 \%$ of patients have a positive family history of dementia $[4,5]$. The three most common genetic causes of FTLD are mutations in the chromosome 9 open reading frame 72 gene (C9orf72), the microtubule-associated protein tau gene (MAPT) and the progranulin gene $(G R N)$ [5]. GRN mutations are found in about 5-10\% of all FTLD cases worldwide [6-8] but have not been reported in the Finnish population thus far [9].

$G R N$ gene is located on chromosome $17 \mathrm{q} 21$. It encodes a precursor protein that can be cleaved to peptides called granulins [8]. Most of the known pathogenic GRN mutations are loss-of-function mutations that cause haploinsufficiency and low plasma progranulin levels $[10,11]$. There are currently almost 150 different mutations and variants reported in the GRN gene (http://www.molgen.ua.ac.be/admutations/). GRN mutations are inherited in an autosomal dominant pattern with incomplete penetrance. The age of onset and the clinical presentation of mutation carriers are variable. It is believed that these are modified by other regulatory mechanisms and risk factors such as allelic variants of the TMEM106B (transmembrane protein 106B) gene [12,13].

$G R N$ is a growth factor involved in multiple physiological processes. Although its exact function in the central nervous system is still unknown it is expressed in neurons and glia cells and is believed to have a role in several processes including inflammation, lysosomal function, neurite outgrowth, response to stress and neuronal WNT signaling [13-15]. Of special interest is its role as a regulator of inflammation. This immunological activity may play a role in neurodegeneration since it was recently shown in Grn -/- mice that progranulin deficiency leads to progressive microgial activation and increased complement production during aging, which gradually leads to synapse pathology [16].

The neuropathology of FTLD is classified into subtypes based on the deposited abnormal protein aggregates. Most of the cases that are tau-negative show immunoreactivity to 
ubiquitin and the majority of those have TDP-43 positive inclusions (FTLD-TDP). FTLDTDP pathology is classified into four subtypes (A-D) that have different distribution types of TDP-43 positive inclusions including neuronal intranuclear inclusions (NIIs), neuronal cytoplasmic inclusions (NCIs), oligodendroglial inclusions and dystrophic neurites (DNs). $G R N$ related FTLD is associated with FTLD-TDP type A, which presents with multiple NCIs, DNs and variable amount of NIIs. The lesions are mostly in layer 2 of the cortex [17-19]. Here we describe a family with a novel GRN mutation p.(Tyr229*). In addition to genetic results, we present the clinical features of the diseased family members and the neuropathological features of the index patient and her nephew.

\section{Materials and methods}

The family pedigree is shown in Figure 1. The index patient was clinically examined and neuropsychological and imaging examinations were performed in Helsinki city hospital. The index patient's nephew and sister were examined in Mikkeli Central Hospital and in HUCH Hyvinkää Hospital respectively. Neuropathological analysis was performed on the index patient and her nephew following standard protocol.

DNA was extracted from the index patient's liver tissue sample and her nephew's formalinfixed paraffin-embedded (FFPE) spleen tissue sample with standard procedures. Participating family members' DNA was extracted from blood leukocytes using standard methods. Whole exome sequencing of the index patient's sample was performed at Institute for Molecular Medicine Finland (FIMM). The target enrichment was performed using Agilent SureSelect Human All Exon V5 kit (Agilent Technologies, Santa Clara, CA, USA). The sequencing was done on a Hiseq2500 platform (Illumina, San Diego, USA) according to manufacturer's protocol. Variant calling was performed using the variant calling pipeline of FIMM, alignment was done to the human genome reference sequence hg19 (GRCh37). Clinical data 
analysis was done with OmnomicsNGS software (Euformatics, Espoo, Finland) focusing on the genes known to cause FTLD. GenBank accession number for the reference sequence used for GRN gene was NM_002087.3.

C9orf72 screening for a possible pathogenic hexanucleotide repeat expansion in the index patient was performed using the repeat-primed PCR method as described by Renton et al.[20].

Sanger sequencing was performed to validate the findings of the whole exome sequencing and to confirm segregation of the mutation with the disease in the family and to study two known TMEM106B gene risk variants in the mutation carriers' samples.

RNA was extracted from two mutation carriers' blood samples with standard procedures. mRNA was reverse-transcribed using the SensiFAST ${ }^{\mathrm{TM}}$ cDNA synthesis kit (BioLine, UK). The GRN expression analysis was performed using commercial TaqMan gene expression assay Hs00963707_g1 (Applied Biosystems) and the CFX96 real-time system (Bio-Rad, Hercules, CA, USA). $\beta$-actin ( $A C T B$ ) was used as endogenous control for normalizing gene expression quantifications. The $G R N$ gene expression levels were expressed as $2^{-\Delta \Delta \mathrm{Ct}}$ fold change values relative to the average of five control samples. All assays were performed using the manufacturer's protocol (Applied Biosystems).

Ethical approval for the study was obtained from the local ethics committee and a written informed consent was obtained from family members and appropriate next of kin of the deceased family members participating in the study. Participating family members received appropriate genetic counselling.

\section{Results}

Clinical and neuropathological features 
The index patient (Individual II-1, Figure 1) was a female who presented with dyspraxia, dysgraphia and dysphasia at the age of 60. She also had mild right sided hemiparesis and symptoms of depression. Her head CT scan showed mild atrophy predominantly on the left upper parietal region and posterior frontal region and a possible infarct behind the left temporal horn. The lateral ventricles were slightly dilated primarily on the left side. Her Mini Mental State Examination (MMSE) score was 28/30. Single-photon emission computed tomography (SPECT) scan at the age of 62 showed a fairly wide hypoperfusion area on the left temporal and parietal regions especially on the anterior part of the temporal lobe. Her symptoms progressed slowly. She had extrapyramidal symptoms and spasticity of the right upper limb. Ultimately she became unable to speak and tetraplegic. Finally, she got pneumonia, and passed away at the age of 78 . Her clinical diagnosis was a progressive degenerative disease, possible corticobasal degeneration.

A neuropathological examination was performed post mortem and revealed dramatic cerebral atrophy. The weight of the brain was $782 \mathrm{~g}$. Both the cerebral cortex and the white matter were severely atrophic, as were the hippocampi, the basal ganglia and the substantia nigrae. Microscopically, there was a dramatic neuronal loss and gliosis in the cortical areas affecting all laminae (Fig. 2A-B) and particularly the deep layers were vacuolated. The cortical structures appeared largely collapsed (Fig 2A), hippocampi were subtotally atrophic, and only the neurons in the dentate fascia and CA4 were preserved. The substantia nigrae showed also moderately severe neuronal loss without Lewy bodies. The white matter was largely atrophic and appeared demyelinated in luxol fast blue stain (Fig 2A), and there were a large number of concentrically hyalinized vessels particularly in the occipital lobe (Fig 2C). There were no neurodegenerative changes in the spinal cord. The muscle sample however, revealed severe atrophic changes, including groups of small angulate fibers, consistent with combined neuronal and inactivity atrophy. 
Immunohistochemistry revealed numerous TDP-43- and p62-positive perinuclear inclusions (Figure 2F) in the dentate fascia of the hippocampus. Furthermore, there was a large amount of cytoplasmic and perinuclear TDP43-positive inclusions in all cortical areas, appearing to be distributed throughout the collapsed cortex (Fig 2E). In addition, a large number of short dystrophic neurites and some intranuclear "cateye"-type inclusions were found (Fig. 2E). Due to severe atrophy, it was not possible to categorize the TDP43-pathology into any of the established categories A-D. Abeta immunostaining showed slight positivity in some hyalinized vessels, and a few diffuse plaques were seen. Tau and $\alpha$-synuclein immunostains were largely negative. The neuropathological diagnosis was FTLD-TDP-43. There were no p62-positive perinuclear inclusions in the granule cells of the cerebellum. The patient's tetraplegia was concluded to be due to the severe cerebral atrophy and no motor neuron disease was diagnosed.

The index patient's nephew (Individual III-2, Figure 1) presented with symptoms of dementia and a change in personality at the age of 60 . His left hand was clumsier and slower. His head MRI scan showed cerebral hemisphere atrophy and also hippocampal atrophy predominantly on the right side. Neuropsychological testing revealed wide-ranging and profound dysfunction predominantly in the frontal and right hemisphere. His clinical diagnosis was frontotemporal dementia. His symptoms progressed quickly and he was bedridden in three years. He had spasticity of the limbs and difficulties in swallowing. He passed away at the age of 63.

At neuropathological examination the weight of the brain was $1150 \mathrm{~g}$. There was considerable cortical atrophy, most predominantly in the frontal and temporal lobes. The basal ganglia were smaller on the right than on the left. Microscopically, neuronal loss and gliosis were found in neocortical areas, particularly on the frontal lobes. The layer II was vacuolated (Fig 2D). There was moderate neuronal loss in the CA1 region and subiculum of the hippocampus. 
The substantia nigrae were preserved. There was only modest hyalination in the vessels of the brain.

The immunohistochemical study showed widespread TDP-43 positive cytoplasmic and perinucear inclusions in the cortical areas, most pronounced in the layer II. A large number of short dystrophic neurites were also seen, consistent with category A -type TDP43- pathology (Fig 2E). In addition, there were some perinuclear TDP43-positive inclusions in the dentate fascia of the hippocampus (Fig 2H). Beta-amyloid immunostain revealed a few neuritic plaques in the hippocampal section. Tau and $\alpha$-synuclein immunostains were considered negative. The neuropathological diagnosis was FTLD-TDP-43.

The index patient's sister (Individual II-5, Figure 1) had previously suffered from depression. She presented with slowly progressing right lower limb weakness and clumsiness at age 68 . She also had periodical right upper limb resting tremor and progressive difficulty in finding words and understanding speech and managing daily activities. Her MMSE score was 21/30. Her head CT scan showed moderately strong general atrophy that was thought to be consistent with vascular atrophy although no focal infarcts were found in the CT scan. The scan showed signs of moderate arteriosclerosis. She was diagnosed clinically with vascular dementia. She developed parkinsonism. She passed away at the age of 73. No neuropathological examination was performed post mortem.

The index patient's brother (individual II-4, Figure 1) had dementia according to family history. He died at the age of 68 , the cause of death was a cerebral infarct. The index patient's father (Individual I-1, Figure 1) who passed away at the age of 64 also had dementia according to family history. His cause of death was bronchopneumonia and cerebral arteriosclerosis. The index patient's mother did not have dementia according to family history. She died at the age of 74. 
Individuals III-1 and III-5 in Figure 1 have not been diagnosed with dementia at the time of this study. They are 57 and 64 years old respectively. Individual III-1 suffers from depression and has had difficulties managing in her job.

\section{Genetic analyses and expression studies}

Whole exome sequencing of the index patient's sample showed a novel heterozygous nonsense $G R N$ mutation c.687T>A, p.(Tyr229*) (Figure 3). The mutation was confirmed by Sanger sequencing. The mutation has not been reported in the 1000 Genomes (http://www.1000genomes.org/) database, in the ExAC database (http://exac.broadinstitute.org/) or in the Exome variant server database (http://evs.gs.washington.edu/EVS/). It has not been reported in the HGMD professional database (http://www.biobase-international.com/product/hgmd). In silico analysis with Mutation Taster (http://www.mutationtaster.org/) predicts the mutation to be disease causing by nonsense mediated mRNA decay.

The GRN mutation appeared to segregate with the disease in the family as it was determined using Sanger sequencing that the index patient's nephew (Individual III-2, Figure 1) also had the familial mutation. There were two yet non-demented mutation carriers identified in the family (Individuals III-1 and III-5, Figure 1). Although there was no tissue sample available for genetic testing from individuals II-4 and II-5 (Figure 1), they were probable carriers of the mutation, because their child (Individuals III-2 and III-5, respectively, Figure 1) and sister (Individual II-1, Figure 1) were carriers of the familial mutation. There were no tissue samples available for genetic testing from the index patient's parents but as her father had dementia according to family history it is possible that he was a carrier of the familial mutation. 
Mutation carriers' blood samples showed decreased mRNA expression, $45 \%$ and $48 \%$ levels as compared to the control individuals (Figure 4) supporting the pathogenicity of the novel mutation.

The index patient did not have a $C 9$ orf72 hexanucleotide repeat expansion mutation. TMEM106B genotyping showed that both the index patient and her nephew (Individuals II-1 and III-2, Figure 1) were homozygous for the risk alleles rs3173615 (C/C) and rs1990622 (T/T). Of the two asymptomatic mutation carriers in Figure 1, individual III-1 was heterozygous for the risk alleles and individual III-5 was homozygous for the protecting alleles rs3173615 (G/G) and rs1990622 (C/C).

\section{Discussion}

We describe a novel $G R N$ mutation in a family with dementia with an autosomal dominant pattern of inheritance. The mutation causes a premature stop codon and is predicted to cause loss-of-function due to nonsense mediated mRNA decay. The mutation appeared to segregate with the disease in the family and a gene expression study showed a decrease in mRNA expression in mutation carriers' samples further supporting the concept of a loss-of-function mutation.

Loss-of-function (and haploinsufficiency) resulting in reduced levels of secreted progranulin protein has been proposed as the most common consequence of $G R N$ mutations [10].

The neuropathological examination of two family members carrying the mutation showed TDP-43 positive inclusions and cerebral atrophy. The index patient's neuropathological findings were extremely severe. The index patient's nephew's neuropathological phenotype was milder and the TDP43 pathology could be classified into subtype A, in accordance with previous reports on $G R N$ mutation carriers. In the index patient, it was not possible to identify the cortical layers due to nearly total loss of neurons, and it was therefore difficult to classify 
the TDP-43 pathology into any of the subtypes A-D. However, it is possible that the TDP43 pathology had represented type A at earlier stages of the disease as the TDP43 pathology consisted of perinuclear, cytoplasmic and intranuclear inclusions in addition to a large number of short dystrophic neurons, but at the end stage the typical predominant localization of the changes in layer II could not be recognized. In addition to severe cortical atrophy and TDP43pathology, the index patient showed severe white matter atrophy and vascular hyalinization, particularly in the occipital region, consistent with a previous MRI report on GRN mutation carriers [21]. Interestingly, a pathological description of SVD-type arterial hyalination has been published in FTLD-tau patients [22].

Clinically the nephew had presented with FTD that had progressed quickly compared to the index patient who had been clinically suspected to have corticobasal syndrome and had a long disease course. The differences between the neuropathology and clinical picture among the family members are consistent with the previous knowledge that $G R N$ mutations can cause variable clinical phenotypes even within the same family. It is also known that there is considerable variation in the age of onset (35-89 years) and in the duration of the illness (3-22 years) [23]. The duration of the illness of the index patient was 18 years, which could be an explanation to the extremely severe neuropathological phenotype in this patient. Most previously described neuropathologies of $G R N$ mutation carriers have been of patients with a much shorter duration of illness [24-26]. The variations in the phenotype even within families have been suggested to be due to other risk factors such as variants in the TMEM106B gene $[12,13]$. Interestingly in this study the index patient and her nephew were both homozygous for the two known TMEM106B gene risk variants although their disease course was markedly different. This suggests other factors besides the TMEM106B gene variants are responsible for the variation in the clinical phenotype in this family. The position of the mutation in the GRN 
gene has not been shown to have a significant effect on the phenotype, which is consistent with the haploinsufficiency mechanism [10,24-26].

To our knowledge this is the first GRN gene mutation reported in the Finnish population. We conclude that the novel GRN mutation c.687T>A (p.Tyr229*) is considered pathogenic by causing nonsense mediated mRNA decay and haploinsufficiency. We recommend genetic counselling to all mutation carriers and their family members.

\section{Acknowledgements}

The authors would like to thank the family for their participation in this study. This work was supported by Helsinki University Hospital (LK, LM, MP, PJT), Academy of Finland (LM), Päivikki and Sakari Solhberg Foundation (LM, PP) and Pirkko and Veikko Mäkelä Foundation (PP).

\section{References}

[1] Rademakers R, Neumann M, Mackenzie IR (2012) Advances in understanding the molecular basis of frontotemporal dementia. Nat Rev Neurol 8, 423.

[2] Bird T, Knopman D, VanSwieten J, Rosso S, Feldman H, Tanabe H, Graff-Raford N, Geschwind D, Verpillat P, Hutton M (2003) Epidemiology and genetics of frontotemporal dementia/Pick's disease. Ann Neurol 54, S29-S31.

[3] Seltman RE, Matthews BR (2012) Frontotemporal Lobar Degeneration: Epidemiology, Pathology, Diagnosis and Management. CNS Drugs 26, 841-870.

[4] Neary D, Snowden J, Mann D (2005) Frontotemporal dementia. Lancet Neurol 4, 771780 .

[5] Bang J, Spina S, Miller BL (2015) Frontotemporal dementia. The Lancet 386, 1672-1682.

[6] Le Ber I, van der Zee J, Hannequin D, Gijselinck I, Campion D, Puel M, Laquerrière A, De Pooter T, Camuzat A, Van den Broeck M, Dubois B, Sellal F, Lacomblez L, Vercelletto M, Thomas-Antérion C, Michel B, Golfier V, Didic M, Salachas F, Duyckaerts C, Cruts M, Verpillat P, Van Broeckhoven C, Brice A (2007) Progranulin null mutations in both sporadic and familial frontotemporal dementia. Hum Mutat 28, 846-855.

[7] Pickering-Brown SM, Rollinson S, Du Plessis D, Morrison KE, Varma A, Richardson AMT, Neary D, Snowden JS, Mann DMA (2008) Frequency and clinical characteristics of 
progranulin mutation carriers in the Manchester frontotemporal lobar degeneration cohort: comparison with patients with MAPT and no known mutations. Brain: A Journal of Neurology 131, 721-731.

[8] Gass J, Cannon A, Mackenzie IR, Boeve B, Baker M, Adamson J, Crook R, Melquist S, Kuntz K, Petersen R, Josephs K, Pickering-Brown SM, Graff-Radford N, Uitti R, Dickson D, Wszolek Z, Gonzalez J, Beach TG, Bigio E, Johnson N, Weintraub S, Mesulam M, White CL, Woodruff B, Caselli R, Hsiung G, Feldman H, Knopman D, Hutton M, Rademakers R (2006) Mutations in progranulin are a major cause of ubiquitin-positive frontotemporal lobar degeneration. Hum Mol Genet 15, 2988-3001.

[9] Krüger J, Kaivorinne A-, Udd B, Majamaa K, Remes AM (2009) Low prevalence of progranulin mutations in Finnish patients with frontotemporal lobar degeneration. European Journal of Neurology 16, 27-30.

[10] Kocerha J, Kouri N, Baker M, Finch N, DeJesus-Hernandez M, Gonzalez J, Chidamparam K, Josephs KA, Boeve BF, Graff-Radford NR, Crook J, Dickson DW, Rademakers R (2011) Altered microRNA expression in frontotemporal lobar degeneration with TDP-43 pathology caused by progranulin mutations. BMC Genomics 12, 527-536.

[11] Finch N, Baker M, Crook R, Swanson K, Kuntz K, Surtees R, Bisceglio G, RoveletLecrux A, Boeve B, Petersen RC, Dickson DW, Younkin SG, Deramecourt V, Crook J, Graff-Radford NR, Rademakers R (2009) Plasma progranulin levels predict progranulin mutation status in frontotemporal dementia patients and asymptomatic family members. Brain: A Journal of Neurology 132, 583-591.

[12] Finch N, Carrasquillo MM, Baker M, Rutherford NJ, Coppola G, DeJesus-Hernandez M, Crook R, Hunter T, Ghidoni R, Benussi L, Crook J, Finger E, Hantanpaa KJ, Karydas AM, Sengdy P, Gonzalez J, Seeley WW, Johnson N, Beach TG, Mesulam M, Forloni G, Kertesz A, Knopman DS, Uitti R, White CL, Caselli R, Lippa C, Bigio EH, Wszolek ZK, Binetti G, Mackenzie IR, Miller BL, Boeve BF, Younkin SG, Dickson DW, Petersen RC, Graff-Radford NR, Geschwind DH, Rademakers R (2011) TMEM106B regulates progranulin levels and the penetrance of FTLD in GRN mutation carriers. Neurology 76, 467-474.

[13] Petkau TL, Leavitt BR (2014) Progranulin in neurodegenerative disease. Trends Neurosci 37, 388-398.

[14] Cruts M, Gijselinck I, Zee J, Engelborghs S, Wils H, Pirici D, Rademakers R, Vandenberghe R, Dermaut B, Martin JJ, van Duijn C, Peeters K, Sciot R, Santens P, De Pooter T, Mattheijssens M, Broeck M, Cuijt I, Vennekens K, De Deyn P, Kumar-Singh S, Van Broeckhoven C (2006) Null mutations in progranulin cause ubiquitin-positive frontotemporal dementia linked to chromosome 17q21. Nature 442, 920-924.

[15] Raitano S, Ordovàs L, De Muynck L, Guo W, Espuny-Camacho I, Geraerts M, Khurana S, Vanuytsel K, Tóth BI, Voets T, Vandenberghe R, Cathomen T, Van Den Bosch L, Vanderhaeghen P, Van Damme P, Verfaillie CM (2015) Restoration of progranulin expression rescues cortical neuron generation in an induced pluripotent stem cell model of frontotemporal dementia. Stem Cell Reports 4, 16-24. 
[16] Lui H, Zhang J, Makinson S, Cahill M, Kelley K, Huang H, Shang Y, Oldham M, Martens L, Gao F, Coppola G, Sloan S, Hsieh C, Kim C, Bigio E, Weintraub S, Mesulam M, Rademakers R, Mackenzie I, Seeley W, Karydas A, Miller B, Borroni B, Ghidoni R, Farese Jr. R, Paz J, Barres B, Huang E (2016) Progranulin Deficiency Promotes Circuit-Specific Synaptic Pruning by Microglia via Complement Activation. Cell 165, 921-935.

[17] Cairns NJ, Bigio EH, Mackenzie IR, Neumann M, Lee VM, Hatanpaa KJ, White CL, Schneider JA, Grinberg LT, Halliday G, Duyckaerts C, Lowe JS, Holm IE, Tolnay M, Okamoto K, Yokoo H, Murayama S, Woulfe J, Munoz DG, Dickson DW, Ince PG, Trojanowski JQ, Mann DM (2007) Neuropathologic diagnostic and nosologic criteria for frontotemporal lobar degeneration: consensus of the Consortium for Frontotemporal Lobar Degeneration. Acta Neuropathol 114, 5-22.

[18] Mackenzie IR, Neumann M, Baborie A, Sampathu DM, Du Plessis D, Jaros E, Perry RH, Trojanowski JQ, Mann DM, Lee VM (2011) A harmonized classification system for FTLDTDP pathology. Acta Neuropathol 122, 111-113.

[19] Lashley T, Rohrer JD, Mead S, Revesz T (2015) Review: An update on clinical, genetic and pathological aspects of frontotemporal lobar degenerations. Neuropathol Appl Neurobiol 41, 858-881.

[20] Renton A, Majounie E, Waite A, Simón-Sánchez J, Rollinson S, Gibbs J , Schymick J, Laaksovirta H, van Swieten J, Myllykangas L, Kalimo H, Paetau A, Abramzon Y, Remes A, Kaganovich A, Scholz S, Duckworth J, Ding J, Harmer D, Hernandez D, Johnson J, Mok K, Ryten M, Trabzuni D, Guerreiro R, Orrell R, Neal J, Murray A, Pearson J, Jansen I, Sondervan D, Seelaar H, Blake D, Young K, Halliwell N, Callister J, Toulson G, Richardson A, Gerhard A, Snowden J, Mann D, Neary D, Nalls M, Peuralinna T, Jansson L, Isoviita V, Kaivorinne A, Hölttä-Vuori M, Ikonen E, Sulkava R, Benatar M, Wuu J, Chiò A, Restagno G, Borghero G, Sabatelli M, Heckerman D, Rogaeva E, Zinman L, Rothstein J, Sendtner M, Drepper C, Eichler E, Alkan C, Abdullaev Z, Pack S, Dutra A, Pak E, Hardy J, Singleton A, Williams N, Heutink P, Pickering-Brown S, Morris H, Tienari P, Traynor B (2011) A Hexanucleotide Repeat Expansion in C9ORF72 Is the Cause of Chromosome 9p21-Linked ALS-FTD. Neuron 72, 257-268.

[21] Caroppo P, Le Ber I, Camuzat A, Clot F, Naccache L, Lamari F, De Septenville A, Bertrand A, Belliard S, Hannequin D, Colliot O, Brice A (2014) Extensive white matter involvement in patients with frontotemporal lobar degeneration: think progranulin. JAMA Neurol 71, 1562.

[22] Thal DR, von Arnim, Christine A. F., Griffin WS, Mrak RE, Walker L, Attems J, Arzberger T (2015) Frontotemporal lobar degeneration FTLD-tau: preclinical lesions, vascular, and Alzheimer-related co-pathologies. J Neural Transm 122, 1007-1018.

[23] van Swieten JC, Heutink P (2008) Mutations in progranulin (GRN) within the spectrum of clinical and pathological phenotypes of frontotemporal dementia. The Lancet Neurology 7 , 965-974.

[24] Mackenzie IRA (2007) The neuropathology and clinical phenotype of FTD with progranulin mutations. Acta Neuropathol 114, 49. 
[25] Mackenzie I.R.A., Baker M., Pickering-Brown S., Hsiung G.-Y.R., Lindholm C., Dwosh E., Gass J., Cannon A., Rademakers R., Hutton M., Feldman H.H. (2006) The neuropathology of frontotemporal lobar degeneration caused by mutations in the progranulin gene. Brain 129, 3081-3090.

[26] Josephs K.A., Ahmed Z., Katsuse O., Parisi J.F., Boeve B.F., Knopman D.S., Petersen R.C., Davies P., Duara R., Graff-Radford N.R., Uitti R.J., Rademakers R., Adamson J., Baker M., Hutton M.L., Dickson D.W. (2007) Neuropathologic features of frontotemporal lobar degeneration with ubiquitin-positive inclusions with progranulin gene (PGRN) mutations. $J$ Neuropathol Exp Neurol 66, 142-151. 


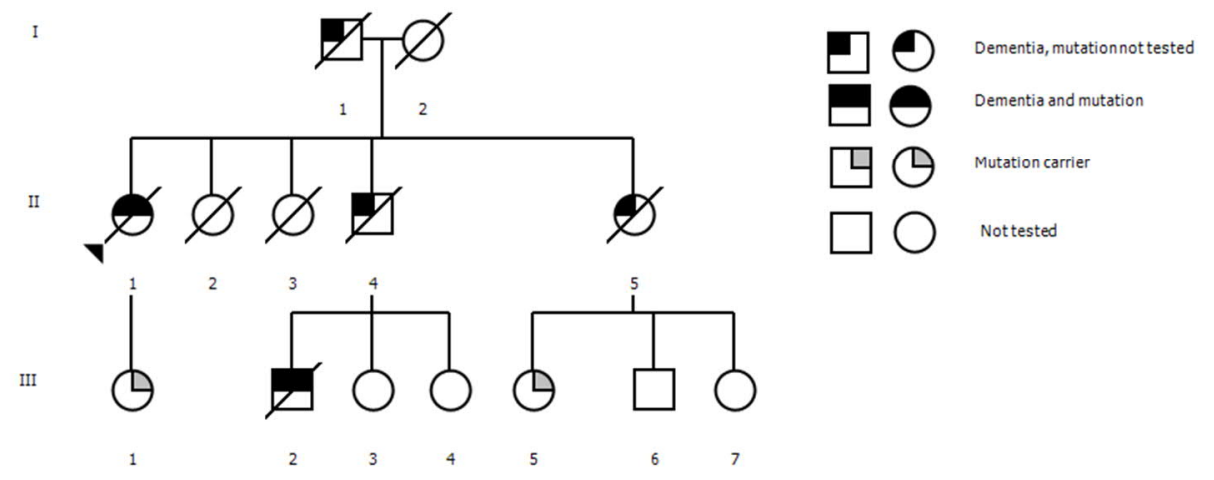

Figure 1. Family pedigree. Deceased individuals are indicated with a diagonal bar. 

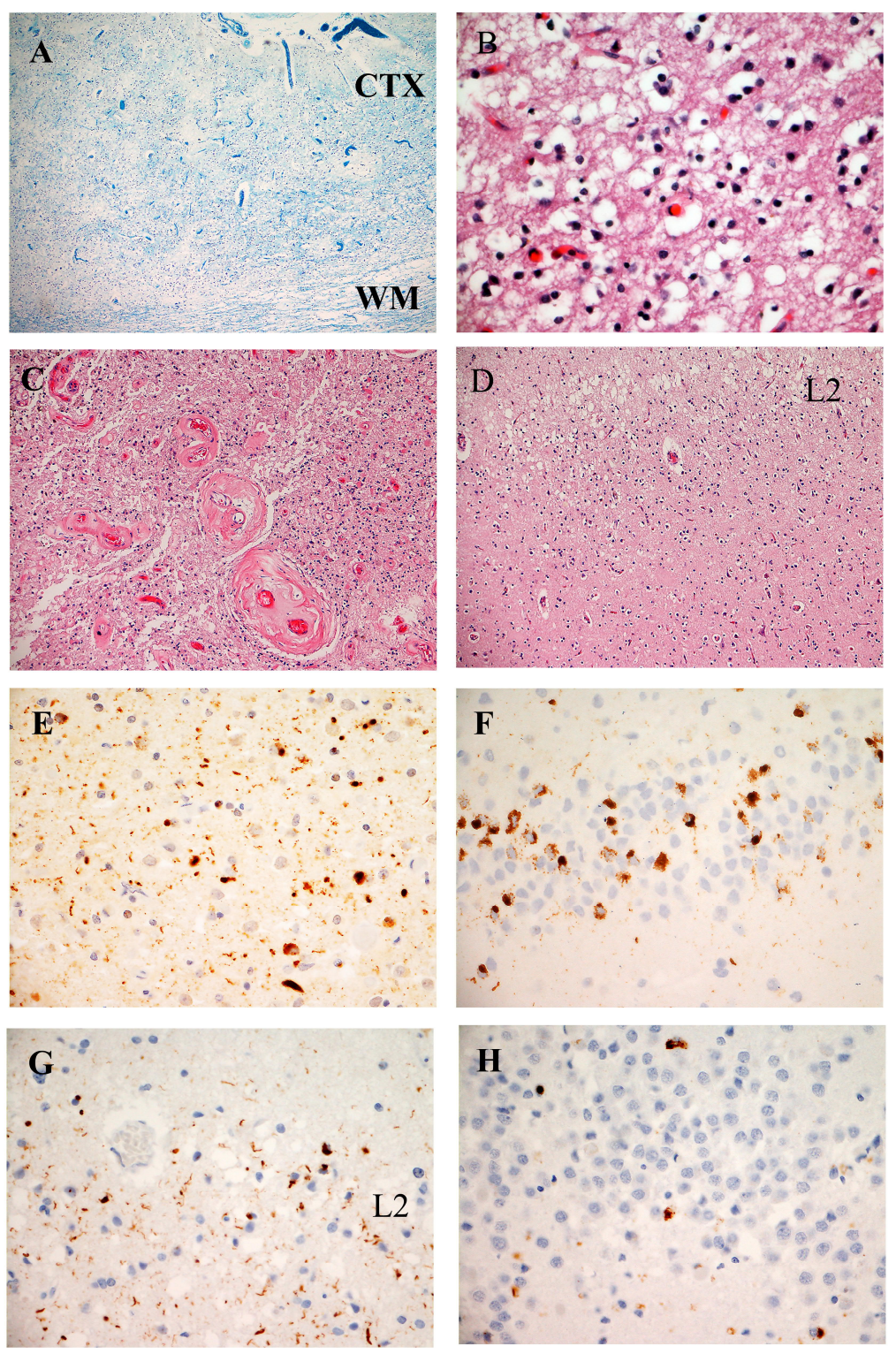

Figure 2. Neuropathological features of the two neuropathologically examined patients. A

The cortical sections of the index patient showed extremely severe neuronal loss, gliosis and vacuolation transcortically. The cortex (CTX) was thinned and atrophic as was the white matter (WM).(occipital cortex 4X magnification, Luxol Fast Blue staining). B Higher magnification shows that there were no or only very few neurons left in the cortex. Severe gliosis and vacuolation was seen (haematoxylin-eosin staining, occipital cortex, $40 \mathrm{X}$ magnification). $\mathbf{C}$ There were many hyalinized vessels in the white matter in the index 
patient's occipital lobe (haematoxylin-eosin staining, occipital lobe, 10X magnification). D In the cortical sections of the index patient's nephew, vacuolation and neuronal loss were most pronounced in the lamina II (L2) (haematoxylin-eosin staining, frontal cortex, 10X magnification). E In the index patient's cortical sections, TDP43-immunostaining revealed pathology throughout the thinned cortex, including cytoplasmic and perinuclear inclusions, a large number of short neurites and some intranuclear inclusions (40X magnification, TDP43 immunostaining, parietal section). F . There were numerous perinuclear TDP-immunoreactive inclusions in the hippocampal dentate fascia of the index patient (40X magnification, TDP43 immunostaining). G. There was abundant TDP-immunopositivity in the nephew's cortical sections, most pronounced in the lamina II (L2) (40 X magnification, TDP43 immunostaining, frontal section). H. The perinuclear inclusions were few in number in the dentate fascia of the nephew (40X magnification, TDP43 immunostaining). 


\section{Tाग}

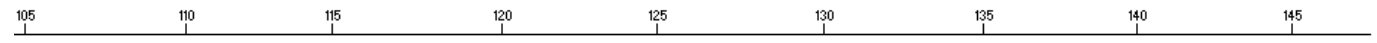

NM_002087.3:c.687T>A, p.(Tyr229*)

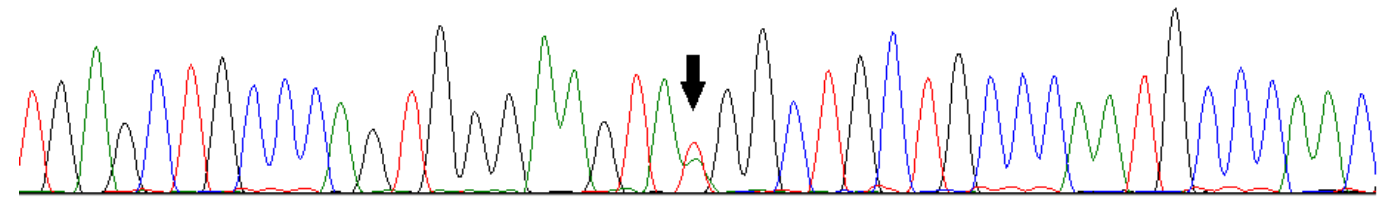

Figure 3. Electropherogram of the GRN mutation c.687T>A, p.(Tyr229*) 


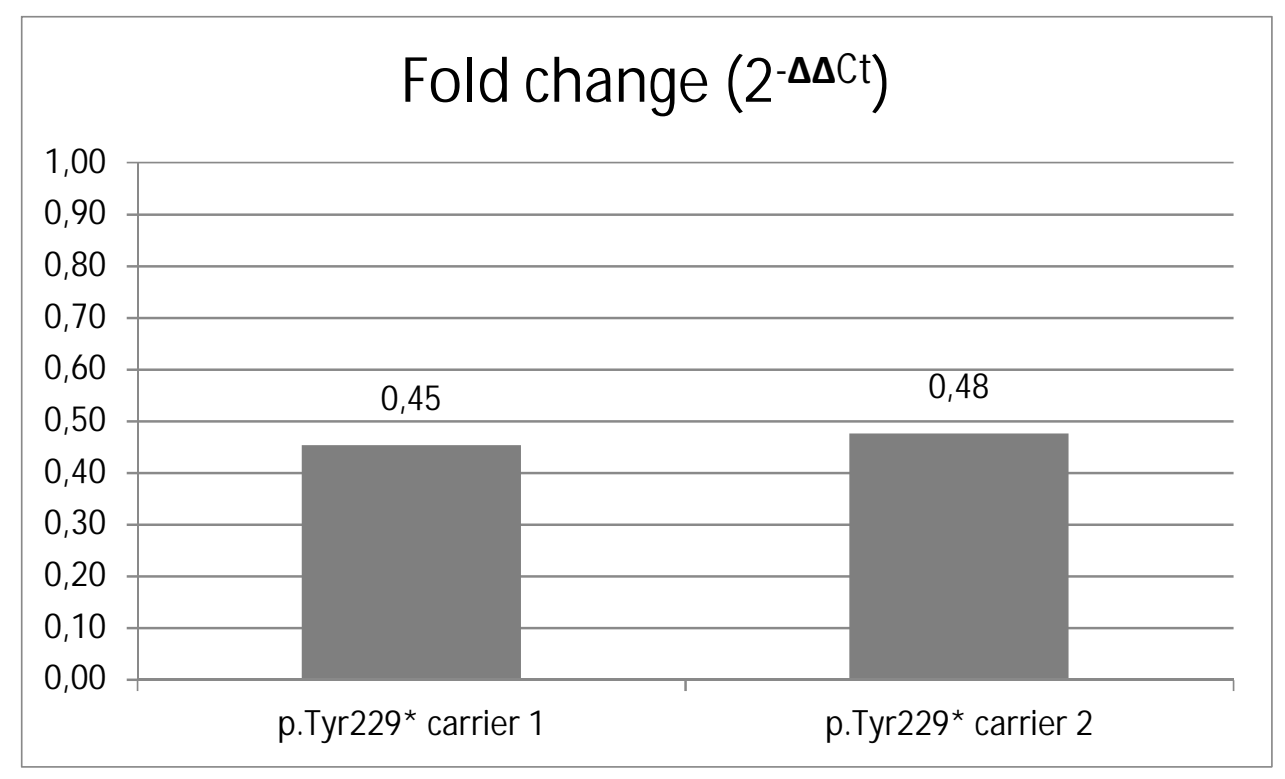

Figure 4. Normalized $G R N$ mRNA expression in two carriers of the p.Tyr229* mutation relative to the average normalized $G R N$ mRNA expression in five unaffected controls. Fold change was calculated with the $2^{-\Delta \Delta \mathrm{Ct}}$ method. 